\title{
Preface to the Special Issue in Memory of Professor Saul Abarbanel
}

\section{Alina Chertock ${ }^{1} \cdot$ Adi Ditkowski ${ }^{2} \cdot$ Anne Gelb $^{3} \cdot$ Sigal Gottlieb $^{4} \cdot$ Semyon Tsynkov ${ }^{1}$}

Published online: 11 November 2019

(c) Springer Science+Business Media, LLC, part of Springer Nature 2019

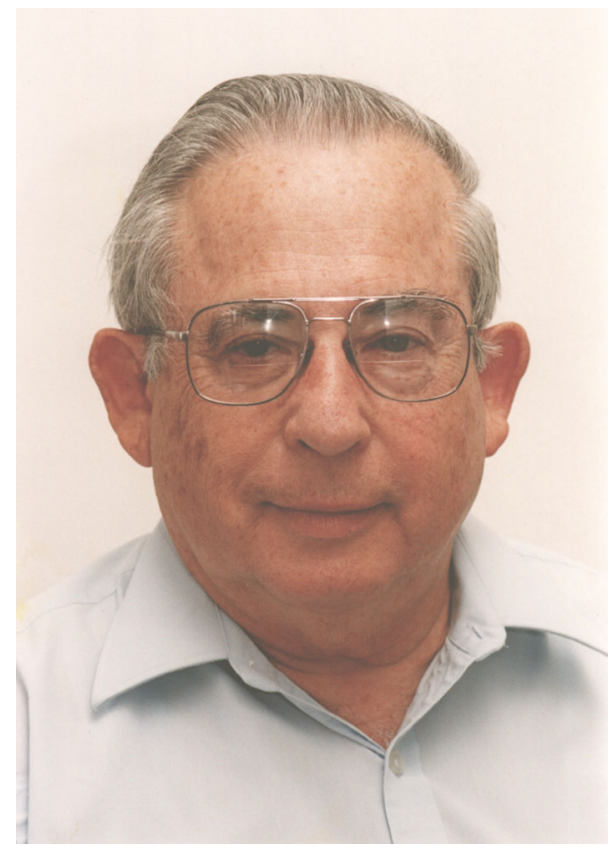

Saul Abarbanel

June 1, 1931-December 22, 2017

\footnotetext{
$凶 \quad$ Sigal Gottlieb

sigalgottlieb@gmail.com

1 North Carolina State University, Raleigh, NC, USA

2 Tel Aviv University, Tel Aviv, Israel

3 Dartmouth College, Hanover, NH, USA

4 University of Massachusetts Dartmouth, Dartmouth, MA, USA
} 
This issue is dedicated to the memory of a prominent Israeli-American applied mathematician Professor Saul Abarbanel who passed away on December 22, 2017, at the age of 86. Throughout his distinguished career, Prof Abarbanel has consistently and passionately emphasized the key role of mathematics in the design of efficient numerical algorithms that can tackle challenging real-world problems. The triad of theory, computation, and applications has been at the cornerstone of all his research endeavors. The papers in this special issue demonstrate the close interaction between these three components and thus commemorate the lifelong contribution by Prof Abarbanel to the discipline of applied mathematics.

Saul Abarbanel was born in 1931 in Montclair, New Jersey, USA. His family moved to Israel when he was very young. According to Saul's own account (provided in an interview conducted by Philip Davis in 2003 [1]), mathematics was his favorite subject when he was a high school student in Tel Aviv. Upon completion of his Israeli military service in 1950, he returned to the United States to study aeronautical engineering at the Massachusetts Institute of Technology. Saul continued at MIT for his graduate studies as well, and in 1959 earned his Doctorate in theoretical aerodynamics with minors in physics and mathematics; his $\mathrm{PhD}$ supervisor was Leon Trilling. However, as early as after the first year of graduate school, Sauls interests started to shift toward applied mathematics, a passion that increased under the mentorship of Norman Levinson. Saul stayed at MIT for one more year after finishing his $\mathrm{PhD}$ and then returned to Israel for a one-year postdoctoral position at the Weizmann Institute of Science. It is during this time that he met his future wife Joan. Although she was from Boston, they had not met during Saul's time at MIT.

Saul became a faculty member at the MIT Department of Aeronautics and Astronautics in 1961, and then returned to Israel in 1964 to become the first Chair of the recently created Department of Applied Mathematics at Tel Aviv University. Tel Aviv University remained Saul's academic home ever since. He held a variety of influential administrative positions throughout his career, including that of Rector of the University (Provost) from 1977 until 1980. He later served as Director of the Raymond and Beverly Sackler Institute of Scientific Computations and the Mortimer and Raymond Sackler Institute of Advanced Studies, both at Tel Aviv University. He was also the IBM Distinguished Visiting Research Professor at Brown University.

In the early part of his career (at MIT and the first years at Tel Aviv University), Saul mainly focused on the modeling and mathematical analysis of problems in the field of heat transfer and fluid dynamics. He was among the pioneers who realized early on the immense potential of numerical computation in studying a broad variety of applied problems. Saul's own research contributions, as well as his leadership, helped integrate advanced numerical methods into science and engineering, which made Tel Aviv University a world class leading institution in applied and computational mathematics.

In the late 1960s, Saul and his students began constructing and analyzing new finite difference approximation methods for partial differential equations. Some of their notable work included the development of algorithms for reducing oscillations near discontinuities (shocks) in the discrete solutions of hyperbolic PDEs, as well as convergence acceleration techniques for the solutions to parabolic PDEs that approach steady state. Saul was among the first computational scientists to promote the use of high order numerical methods for computing solutions to PDEs. He developed compact high order accurate finite difference schemes to approximate initial boundary value problems for hyperbolic systems and obtained key results on their stability. In his 2003 interview conducted by Philip Davis [1], Saul indicated that perhaps one of his most fundamental contributions was the 1981 paper (co-authored with David Gottlieb) on symmetrization of the linearized Navier-Stokes equations (simultaneous symmetrization of all coefficient matrices), because it paved the way in applying stability 
theory that could not have otherwise been applied. This paper also inspired the subsequent momentous work by Ami Harten on computing the weak solutions for nonlinear hyperbolic equations. Saul made another important contribution in the development and analysis of artificial boundary conditions and perfectly matched layers for the numerical solutions of exterior problems, in particular, those for Maxwells equations. He conducted the first ever systematic study of well-posedness for various PMLs (again in collaboration with David Gottlieb) that provided a theoretical foundation for a large number of subsequent research investigations in computational electromagnetism. Saul's final paper [2] was published when he was 84 years of age, and he remained active until his very last days.

Saul's influence extended far beyond Tel Aviv University, and he was internationally recognized for his leadership. He served both as Chairman of the Coordinating Committee of University Heads as well as Chairman of the National Research and Development Council in Israel. He also served as the Israeli Observer (on behalf of the National Academy of Sciences) at the European Science Research Councils (ESRC) of the European Science Foundation and as Chair of the Applied Mathematics Committee in the European Mathematical Society. He twice held the post of Chair of the Council of Institutions of the Universities Space Research Association (USRA) in the United States. For many years he was affiliated as consultant with the Institute for Computer Applications in Science and Engineering (ICASE), NASA Langley Research Center in Hampton, VA, USA.

ICASE was one of the preeminent places for scientific computation from the 1970s through the 1990s. Its distinct culture fostered interactions and collaboration between NASA scientists and ICASE staff and visitors. The Institute played a special role in Saul's life as he was one of its longest serving members. In the 2018 paper [3] given at the Future CFD Technologies Workshop, Manny Salas, ICASE Director between 1996 and 2002, wrote that "A great deal of the ICASE research was conducted by visiting scientists on one of the many oversize blackboards in the ICASE conference room. Here visiting scientists, Saul Abarbanel was a prominent practitioner of this art, would discuss their research topic with whoever was at ICASE at the time or whoever just happened to walk by the conference room." And the epigraph to the same 2018 paper is a 1997 quote from Saul: "For the past 25 years ICASE has been one of the very few places where one could engage in "strategic" research-the type of scientific inquiry which has all the attributes of basic research but which has an application horizon in the not-very-far future. As a result, ICASE has left its mark in many areas of aerospace applications."

Saul educated generations of students at Tel Aviv University. He taught classes alongside with maintaining a research program at the highest level, having important administrative responsibilities, and serving in the various leadership positions. He liked interacting with students in the classroom and kept teaching PDEs and Fluid Mechanics long after he retired from regular faculty and became Professor Emeritus. Saul has also provided training and mentorship to a large number of younger researchers-doctoral students and postdocs. Many of his former trainees went on to become leading international figures in their respective research disciplines.

In addition to running an extensive research program and revolving in high orbits of administration and leadership, Saul was well known for his wonderful sense of humor. He displayed funny mathematics cartoons on the door of his office in the Schreiber Building of Tel Aviv University. Knocking on his door was always followed by pleasant and enlightening conversation. He was calm and even-tempered. He liked to say that when he quits doing mathematics (which never happened, of course) he would become a cab driver, because the crazy traffic in Tel Aviv wouldnt really get under his skin. To this day, Saul's colleagues and friends like to retell the legendary Abarbanel jokes. Jeff Danowitz, a former PhD student of 
Saul's, recalls an episode when a dog came into the classroom where Saul was teaching a class in PDEs. It ran around knocking everything down and eventually sat down in a chair! Saul began the lecture as if nothing was going on although everyone was laughing at the dog sitting in a chair. Someone asked him "Don't you see theres a dog in the room? That's why we're laughing." Saul replied in a serious tone, "OK. He's sitting down. Apparently, he wants to learn partial differential equations. How about you?"

Undoubtedly, the capacity to communicate clearly is a positive indication of the clarity of one's thought. Saul possessed these qualities to the highest degree possible. Not only his professional peers, but the people with whom he interacted socially have always been impressed by how transparent even the most complex things become under Saul's explanation. He had many friends throughout the world, and well beyond his professional circle. He liked to joke that one does not need to exercise influence if there are the right connections. The extent of his own connections was unparalleled, which he was always willing to use to help the people around him.

Two research meetings in memoriam of Prof Saul Abarbanel were organized in 2018 by his former colleagues and students: the workshop Advances in PDEs: Theory, Computation, and Applications to CFD was held at the NSF-funded Institute for Computational and Experimental Research in Mathematics (ICERM) in August 2018 [4], and the international conference Advances in Applied Mathematics at Tel Aviv University in December 2018. The summary of Saul's scientific accomplishments for this Foreword was partially derived from the lecture given by Bertil Gustafsson at the workshop hosted by ICERM in Providence, Rhode Island, USA [5]. This workshop was attended by over 120 people, most of the talks are available online and some of the papers in this issue were presented by speakers at this workshop. Other papers in the current special issue were presented at the meeting in Tel Aviv, which was sponsored by the European Office of Aerospace Research and Development (United States Air Force Office of Scientific Research), ${ }^{1}$ United States Army Research Office, ${ }^{2}$ Tel Aviv University, North Carolina State University, Israeli Mathematical Union, and Mathematical Institute at Tel Aviv. The meeting featured twenty five invited speakers from different countries. It has also included the first award ceremony for the newly established Abarbanel Prize in Applied Mathematics and a memorial session where presenters shared their memories about Saul as a scientist, colleague, teacher, mentor, leader, administrator, and a delightful human being. Both conferences reflected the overall appreciation and high esteem for which Saul Abarbanel was held by the international applied mathematics research community.

\section{References}

1. Davis, P.: An interview with Saul Abarbanel, on July 29. SIAM oral histories project. (2003) http://history. siam.org/pdfs2/Abarbanel-complete.pdf

2. Abarbanel, S., Ditkowski, A.: Wave propagation in advected acoustics within a non-uniform medium under the effect of gravity. Appl. Numer. Math. 93, 61-68 (2015)

3. Salas, M.: paper given in Future CFD Technologies Workshop. https://scientific-sims.com/cfdlab/ WORKSHOP/workshop.html

1 The authors gratefully acknowledge the support of the United States Air Force Office of Scientific Research European Office of Aerospace Research and Development Grant Number FA9550-19-1-7001

2 The authors gratefully acknowledge the support of the United States Army Research Office Grant Number W911NF-18-1-0294 
4. ICERM workshop on "Advances in PDEs: Theory, Computation and Application to CFD", The authors gratefully acknowledge the support of ICERM, under the National Science Foundation Grant No. DMS1439786. https://icerm.brown.edu/topical_workshops/tw18-6-apde/

5. Gustafsson, B.: Saul Abarbanel-half a century of scientific work, at ICERM workshop on "Advances in PDEs: Theory, Computation and Application to CFD". https://icerm.brown.edu/video_archive/?play=1658

Publisher's Note Springer Nature remains neutral with regard to jurisdictional claims in published maps and institutional affiliations. 